\title{
Ein Beispiel einer Blended-Learning-Veranstaltung
}

\section{Investition und Finanzierung als Online-Vorlesung}

\author{
Andreas Löffler
}

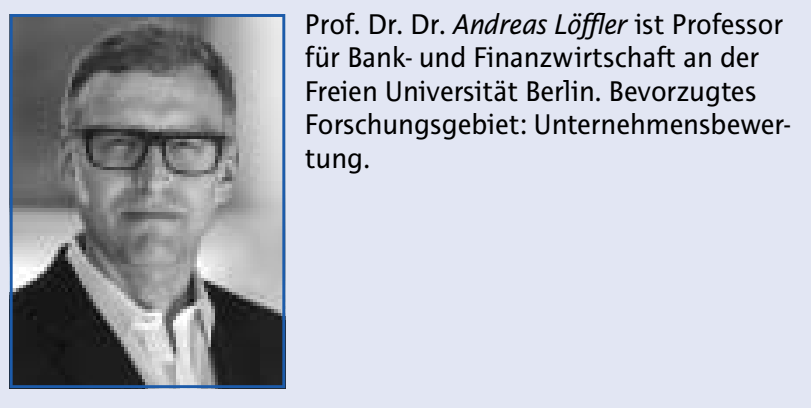

Seit mehreren Jahren wird eine Massenveranstaltung des 2. Semesters (Bachelor BWL bzw VWL) an der Freien Universität mit den Mitteln des Blended-Learning angeboten. In meinem Vortrag berichte ich von den technischen Voraussetzungen, den pädagogischen Herausforderungen und der studentischen Rezeption dieser Veranstaltung.

Stichwörter: Blended-Learning, Online-Vorlesung, eLearning

\section{Massenveranstaltungen als Herausforderung in der Lehre}

Viele der jetzt tätigen Hochschullehrerinnen und Hochschullehrer sind in einer Zeit groß geworden, in der ein Studium an einer Universität einen gewissen Seltenheitswert hatte. Beispielsweise konnten nur sehr wenige Mitschüler des Autors in den 80er Jahren des vergangenen Jahrhunderts das Abitur ablegen, weil der volkswirtschaftliche Bedarf an Akademikerinnen und Akademikern „überschaubar" war. Diese Zeiten sind heute vorbei. Im WS 2014/15 meldete das Statistische Bundesamt über 800.000 Studienanfängerinnen und Studienanfänger und es ist nicht davon auszugehen, dass in den nächsten Jahren diese Zahlen (trotz demografischem Knick) wesentlich sinken werden. Das hat Folgen für die Lehre an den Universitäten und Fachhochschulen.

Oft werden dabei Blended-Learning und eLearning erwähnt. Und auf den ersten Blick erscheint eine OnlineVorlesung als das Mittel der Wahl, um den Massenansturm von Studenten zu bewältigen. Ist sie einmal aufgezeichnet, könnte man sie nicht nur für 100, sondern auch für 500 oder gar 1.000 Studentinnen verwenden - die Veranstaltung wirkt beliebig skalierbar. Der Autor dieses Beitrages hat seit einer Dekade Erfahrung mit derartigen Ver- anstaltungen und bietet seit drei Jahren das Modul Investition und Finanzierung an der Freien Universität als Blended-Veranstaltung an; daher scheint es sinnvoll, ein erstes Fazit dieses Instrumentes zu ziehen.

An der Vorlesung nehmen jedes Jahr etwa 450 bis 500 Personen das erste Mal teil, sie findet (noch) im größten Hörsaal der Fakultät statt. Sie richtet sich ausschließlich an Studentinnen und Studenten der BWL und VWL, Nebenhörer anderer Fächer oder Gasthörer sind nicht anwesend. Es handelt sich um eine Bachelor-Veranstaltung des zweiten Semesters, das Curriculum dürfte dem jeder anderen deutschen Universität entsprechen.

\section{Technische und organisatorische Voraussetzungen}

Früher benötigte man für eine klassische Vorlesung nur eine Tafel und eine Packung Kreide. Für eine Online-Veranstaltung sind dagegen substantielle Anfangsinvestitionen vonnöten. Üblicherweise wird man die Vorlesung aufzeichnen und dann auf einem Online-Portal bereitstellen wollen. Die dafür notwendigen Komponenten unterteilen sich in Software und Hardware. Kaum überraschend dürfte sein, dass dabei die Hardware den teuersten Teil bildet. Wer jemals die schnell und preiswert bereitgestellten Videos auf Youtube, Vimeo \& Co. angeschaut hat, weiß, dass man ohne ein professionelles Mikrofon keine seriöse Aufzeichnung, die sich eine Studentin länger als zehn Minuten anschauen will, bekommt. Wer sich beim Vortrag auch noch bewegt (was die meisten Hochschullehrer tun), muss mindestens 600 Euro für ein tragbares professionelles Mikrofon einplanen.

Die Aufzeichnung der eigenen Person (mit Webcam oder Kamera) erscheint dem Autor dabei eher entbehrlich. Studenten empfanden es als viel gravierender, wenn der Ton von mittlerer bis schlechter Qualität war. Fehlende Bildaufzeichnungen wurde nie bemängelt, teilweise sogar begrüßt: Dadurch wurde die Fläche, den die dargestellten Folien einnahmen, größer.

Der Autor hat die Vorlesung an einem so genannten Smartboard aufgezeichnet. Dies kann man sich als sehr großes Tablett $(1,5 \mathrm{~m} \times 1 \mathrm{~m})$ vorstellen, das berührungsempfindlich ist und ohne separaten Beamer auskommt, aber jeden Desktop eines handelsüblichen PCs vollständig und 1:1 abbilden kann. Derartige Geräte sind nach wie vor sehr teuer und die Anschaffung wird wahrscheinlich nur 
bei externer Finanzierung möglich sein, oft leistet sich eine Fakultät nur ein einziges Gerät. Zudem sind Smartboards sehr wartungsintensiv, auch ein Diebstahlschutz sollte bedacht sein. Sie haben aber den Vorteil, dass die Dozentin auch in der Aufzeichnung ein möglichst authentisches Vorlesungsgefühl vermitteln kann (man spricht an einer Tafel, auf der man mit virtueller Kreide schreiben und wischen kann) und es ist möglich, diverse andere Softwareprogramme wie Excel und Stata spielend in die Präsentation einzubinden. Allerdings muss die Bedienung eines solchen Smartboads geübt werden, das Display reagiert auf jede Berührung und nicht selten geschieht nicht immer das, was die Dozentin gerade wollte. Während einer Aufzeichnung kann dies enorm störend wirken.

Als Software empfiehlt sich ein Standardprodukt, mit Camtasia beispielsweise kann man für weniger als 300 Euro den Bildschirminhalt mitschneiden, das Tonsignal darüberlegen und, wenn gewünscht, das Bild einer Webcam ebenfalls der Aufzeichnung hinzufügen. Camtasia erlaubt ebenfalls, Frage-Antwort-Elemente (,Quiz“) in die Videos einzubauen und erleichtert die Bearbeitung der Aufzeichnungen ungemein. Der Export des fertigen Produktes geschieht mit wenigen Klicks, die Videos können dann auf verschiedenen Smartphones (eventuell nach Installation kostenloser Apps) oder einem Browser sofort betrachtet werden.

Neben den unmittelbar finanziellen Kosten sollte aber der personelle Aufwand keinesfalls unterschätzt werden. Abstürze, Softwarefehler, Bedienungsfehler, Darstellungsschwierigkeiten wegen unterschiedlicher Displayauflösungen und ähnliche Probleme sind immer noch an der Tagesordnung. Mit der derzeitigen Technik muss man damit rechnen, dass die Aufzeichnung einer einstündigen Vorlesung eine Nachbearbeitung von etwa zwei bis drei Stunden notwendig macht. Von daher verbietet sich die Einschätzung, die Online-Vorlesungen seien eine Art Allheilmittel, um den Massenansturm an Universitäten zu bewältigen: Zuerst einmal sind (gute) Online-Vorlesungen teuer! Vor dem Hintergrund der Tatsache, dass in einigen Landes-Lehrverpflichtungsverordnungen Online-Veranstaltungen eher stiefmütterlich behandelt werden, sollte man sich den Aufwand einer solchen eLearning-Veranstaltung reiflich überlegen.

Ebenso sollte man nicht unterschätzen, dass die Aufzeichnung einer Veranstaltung ein anderes Lehrverhalten bedingt. Wer möchte, dass die Vorlesungen über zwei oder mehr Semester genutzt wird, sollte tagesaktuelle Bezüge vermeiden - diese wirken in zwei Jahren sicherlich deplatziert. Auch führt nach Einschätzung des Autors die Möglichkeit, das gesprochene Wort detailliert nachhören zu können, dazu, dass man eher einen farbloseren Stil, in jedem Fall eine politisch viel korrektere Sprache verwendet. Flapsige Bemerkungen jedenfalls, die dazu dienen, ein Auditorium aus der Müdigkeit zu wecken, könnten, wenn online gestellt, Anlass zu Beschwerden geben.

Will man Vorlesungen aufzeichnen, kann dies mit und ohne Publikum geschehen. Wer mit Publikum aufzeich- net, sollte bei Nachfragen aus dem Auditorium unbedingt ein Mikrofon (und dann auch ein spezielles Mischpult, das eventuell verschiedene Mikrofontypen auch koppeln kann) nutzen - derartige Fragen sind auf einem Mitschnitt sonst praktisch nie zu verstehen und die dann gegebenen Antworten für den Zuschauer wertlos. Dass damit der technische Aufwand weiter steigt, sollte klar sein. Alternativ bietet es sich an, die Vorlesung gänzlich ohne Publikum aufzuzeichnen. Hier entsteht aber ein neues Problem: Während es in einer Vorlesung laut wird, wenn man zu ungenau erklärt oder die Darstellung zu schwierig und komplex wird, fehlt eine derartige Rückkopplung bei einer Aufzeichnung ohne Publikum völlig. Daher empfiehlt es sich, eine Vorlesung erst dann ohne Zuhörerinnen und Zuhörer aufzuzeichnen, wenn man selbst den Stoff und die didaktischen Elemente mehrfach ausprobiert hat und die Klippen, die sich Studentinnen bieten, ausreichend kennt. Ein weiterer Effekt von aufgezeichneten Vorlesungen besteht darin, dass in einer Stunde Online-Vorlesung nahezu immer (viel) mehr Stoff vermittelt wird als in einer Stunde Präsenzveranstaltung. Von daher besteht bei reinen Online-Vorlesungen die Gefahr, viel mehr Stoff vermitteln zu wollen als bei einer Präsenzvorlesung.

Es sollte auch klar sein, dass sich nicht jeder Vorlesungsinhalt für eine Aufzeichnung und Mehrfachnutzung anbietet. Wer heute eine Vorlesung zum aktuellen Steuerrecht mitschneidet, muss schon eine gehörige Portion Vertrauen in den Fiskus haben, wenn er sie ein Jahr später immer noch verwenden will.

Zuletzt benötigt man Webspace, auf dem die Veranstaltung online gestellt wird. Da heute an allen Hochschulstandorten entsprechende Streamer verfügbar sind (oder in Lernplattformen wie Elias und Blackboard integriert werden können), sollte dies kein Problem mehr sein. Auch dürfte der von Lernvideos ausgehende Webtraffic keine nennenswerte Belastung mehr darstellen.

\section{Studentische Reaktionen}

Der Autor verwendet neben den Online-Vorlesungen Investition und Finanzierung weitere Lernmaterialien, darunter ein Skript als auch mehrere Übungsaufgaben. Diese Übungsaufgaben sind elektronisch verfügbar; trägt man die Lösung ein, so wird einem sofort die Musterlösung angezeigt. Das Lösen der Übungsaufgaben wird bei der Prüfungsleistung (Klausur) nicht berücksichtigt, weil die rechtlichen Hürden viel zu hoch sind. Neben reinen Online-Vorlesungen gibt es einzelne Präsenzveranstaltungen am Ende des Semesters, in denen Anwendungen des gelehrten Wissens (bis hin zu praktischen Fällen) diskutiert werden.

Da die genutzte Software ein Tracking der studentischen Nutzung erlaubt, konnte das Lernverhalten etwas genauer unter die Lupe genommen werden. Weitere Hinweise ergaben die Lehrevaluationen, die an der Fakultät regelmäßig durchgeführt werden. 